\title{
Glassy behavior of electrons near metal-insulator transitions
}

\author{
V. Dobrosavljević, D. Tanasković, and A.A. Pastor \\ Department of Physics and National High Magnetic Field Laboratory \\ Florida State University, Tallahassee, Florida 32306
}

(Dated: October 28, 2018)

\begin{abstract}
The emergence of glassy behavior of electrons is investigated for systems close to the disorder and/or interaction-driven metal-insulator transitions. Our results indicate that Anderson localization effects strongly stabilize such glassy behavior, while Mott localization tends to suppress it. We predict the emergence of an intermediate metallic glassy phase separating the insulator from the normal metal. This effect is expected to be most pronounced for sufficiently disordered systems, in agreement with recent experimental observations.
\end{abstract}

PACS numbers: $71.30 .+\mathrm{h}, 72.15 . \mathrm{Rn}, 71.27 .+\mathrm{a}$

In many disordered electronic [1] systems, electronelectron interactions and disorder are equally important, and lead to a rich variety of behaviors which remain difficult do understand. Their competition often leads to the emergence of many metastable states and the resulting history-dependent glassy dynamics of electrons. Such behavior has often been observed in sufficiently low mobility materials [2, 3], but more recent experiments [4, 5] have provided striking and precise information of such non-ergodic dynamics.

Theoretically, the possibility for glassy behavior in the charge sector has been anticipated a long time ago 6] in situations where the electrons are strongly localized due to disorder. In the opposite limit, for well delocalized electronic wavefunctions, one expects a single well defined ground state and absence of glassiness. The behavior in the intermediate region has proved more difficult to understand, and at present little is known as to the precise role and stability of the glassy phase close to the metal-insulator transition (MIT) []]. On physical grounds, one expects the quantum fluctuations [8] associated with mobile electrons to suppress glassy ordering, but their precise effects remain to be elucidated. Note that even the amplitude of such quantum fluctuations must be a singular function of the distance to the MIT, since they are dynamically determined by processes that control the electronic mobility.

To clarify the situation, the following basic questions need to be addressed: (1) Does the MIT coincide with the onset of glassy behavior? (2) How do different physical processes that can localize electrons affect the stability of the glass phase? In the following, we provide simple and physically transparent answers to both questions. We find that: (a) Glassy behavior generally emerges before the electrons localize; (b) Anderson localization [9] enhances the stability of the glassy phase, while Mott localization [7] tends to suppress it. We thus predict the emergence of an intermediate metallic glass phase separating the ordinary metal from the insulator. However, we expect this effect to be of relevance only for sufficiently strong disorder, consistent with recent experiments [4].
As a simplest model where both Anderson and Mott routes to localization can occur, we consider an extended Hubbard model of disordered spin 1/2 electrons, as given by the Hamiltonian

$H=\sum_{i j \sigma}\left(-t_{i j}+\varepsilon_{i} \delta_{i j}\right) c_{i, \sigma}^{\dagger} c_{j, \sigma}+U \sum_{i} n_{i \uparrow} n_{i \downarrow}+\sum_{i j} V_{i j} \delta n_{i} \delta n_{j}$.

Here, $\delta n_{i}=n_{i}-\left\langle n_{i}\right\rangle$ represent local density fluctuations ( $\left\langle n_{i}\right\rangle$ is the site-averaged electron density), $U$ is the onsite interaction, and $\varepsilon_{i}$ are Gaussian distributed random site energies of variance $W^{2}$. In order to allow for glassy freezing of electrons in the charge sector, we introduce weak inter-site density-density interactions $V_{i j}$, which we also also choose to be Gaussian distributed random [10] variables of variance $V^{2} / z(z$ is the coordination number). We emphasize that, in contrast to previous work [8], we shall keep the coordination number $z$ finite, in order to allow for the possibility of Anderson localization. To investigate the emergence of glassy ordering, we formally average over disorder by using standard replica methods [8], and introduce collective $Q$-fields to decouple the inter-site $V$-term [8]. As a result, the replicated partition function can be written in the form

$$
Z^{n}=\int \prod_{i} D c_{i}^{\dagger} D c_{i} D Q_{i} d \varepsilon_{i} P\left(\varepsilon_{i}\right) \exp \{-S\}
$$

where $S=S_{e l}+S_{Q}+S_{i n t}$, with

$$
\begin{aligned}
S_{e l} & =\sum_{i j \sigma} \sum_{a} \int_{0}^{\beta} d \tau c_{i \sigma}^{\dagger a}(\tau)\left[\left(\partial_{\tau}-\mu+\varepsilon_{j}\right) \delta_{i j}-t_{i j}\right] c_{j \sigma}^{a}(\tau) \\
& +U \sum_{a} \int_{0}^{\beta} d \tau c_{i \uparrow}^{\dagger a}(\tau) c_{i \uparrow}^{a}(\tau) c_{i \downarrow}^{\dagger a}(\tau) c_{i \downarrow}^{a}(\tau), \\
S_{Q} & =\frac{1}{2} V^{2} \sum_{i j, a b} \int_{0}^{\beta} \int_{0}^{\beta} d \tau d \tau^{\prime} Q_{i}^{a b}\left(\tau-\tau^{\prime}\right) K_{i j} Q_{j}^{a b}\left(\tau-\tau^{\prime}\right),
\end{aligned}
$$


with

$$
S_{\text {int }}=-\frac{1}{2} V^{2} \sum_{i, a b} \int_{0}^{\beta} \int_{0}^{\beta} d \tau d \tau^{\prime} \delta n_{i}^{a}(\tau) Q_{i}^{a b}\left(\tau-\tau^{\prime}\right) \delta n_{i}^{b}\left(\tau^{\prime}\right) .
$$

Here, $c_{j \sigma}^{a}\left(\omega_{m}\right)$ are the replicated Grassmann fields 8] corresponding to electrons, $\delta n_{i}^{a}(\tau)=$ $\sum_{\sigma}\left[c_{i \sigma}^{\dagger a}(\tau) c_{i \sigma}^{a}(\tau)-<c_{i \sigma}^{\dagger a}(\tau) c_{i \sigma}^{a}(\tau)>\right], a=1, \ldots n$ $(n \rightarrow 0)$ are the replica indices, and $K_{i j}=\frac{2}{z} f_{i j}^{-1}$ is the inverse lattice matrix corresponding to the interactions $V_{i j}$.

Saddle-point theory. To investigate the glassy behavior on a mean-field level [8], we formally integrate out the conduction electrons to produce an effective action $S_{\text {eff }}[Q]$ for the $Q$-fields, the variation of which produces the following saddle-point conditions

$$
\begin{aligned}
& Q_{i}^{a a}\left(\tau-\tau^{\prime}\right)=\chi\left(\tau-\tau^{\prime}\right)=\left\langle\delta n_{i}^{a}(\tau) \delta n_{i}^{a}\left(\tau^{\prime}\right)\right\rangle_{S P}, \\
& Q_{i}^{a b}\left(\tau-\tau^{\prime}\right)=q^{a b}=\left\langle\delta n_{i}^{a}(\tau) \delta n_{i}^{b}\left(\tau^{\prime}\right)\right\rangle_{S P} ;(a \neq b) .
\end{aligned}
$$

Physically, $\chi\left(\tau-\tau^{\prime}\right)$ is the averaged local dynamic compressibility, and $q^{a b}$ is related to the familiar EdwardsAnderson order parameter [8]. In these expressions, the averages are taken with respect to the saddle-point action for conduction electrons, as given by

$$
S_{s p}=S_{e l}\left[c_{i}^{\dagger}, c\right]+S_{i n t}\left[c_{i}^{\dagger}, c ; \chi, q^{a b}\right]
$$

Glass transition. In our approach, the emergence of many metastable states corresponding to glassy ordering is identified as a replica symmetry breaking (RSB) instability [8]. To perform such a stability analysis, we evaluate the $S_{\text {eff }}[Q]$ at the saddle point, and then examine its variation to infinitesimal RSB perturbations of the form $q^{a b}=q+\delta q^{a b}$. The corresponding stability matrix can be expressed through appropriate correlation function of density fluctuations, and the rest of the analysis is identical as in the classical case [8, 11]. The resulting instability criterion, which corresponds to the vanishing of the relevant eigenvalue of the stability matrix, takes the form

$$
\lambda_{o}=1-V^{2} \sum_{j}\left[\chi_{i j}^{2}\left(\omega_{n}=0\right)\right]_{d i s}=0 .
$$

Here, the non-local static compressibilities are defined (for a fixed realization of disorder) as

$$
\chi_{i j}\left(\omega_{n}=0\right)=-\partial n_{i} / \partial \varepsilon_{j}
$$

where $n_{i}$ is the local expectation value of the electron density, and $[\cdots]_{\text {dis }}$ represents the average over disorder.
The eigenvalue $\lambda_{o}$ play the same role in our theory as the parameter $r$ in a conventional Landau-Ginzburg action, the vanishing of which indicates an ordering instability in the appropriate channel. Such parameters are typically assumed to be smooth (regular) functions of control parameters such as temperature or the Fermi energy. In our case the situation is more interesting: we will show that the correlation function $\chi^{(2)} \equiv \sum_{j}\left[\chi_{i j}^{2}\right]_{d i s}$ is a singular function, diverging at an Anderson-like metalinsulator transition. In this way, Anderson localization can be regarded as a singular perturbation in the case of the glassy ordering of electrons. We should emphasize that this unusual sensitivity to Anderson localization is not found in cases of more conventional transitions to uniform ordering. Our analysis can be easily repeated in such situations, and the corresponding instability criterion would instead involve the average compressibility evaluated at the relevant ordering wave vector $\vec{K}$, as given by

$$
\chi^{(1)}(\vec{K})=\sum_{j} e^{i \vec{K} \cdot \vec{j}}\left[\chi_{i j}\left(\omega_{n}=0\right)\right]_{d i s} .
$$

It is well known [1] that such quantities remain finite (see also below) and thus non-singular at an Anderson transition. Similar behavior is found in the case of a superconducting instability in presence of localization [12]. In this case, the relevant pairing susceptibility (in the Cooper channel) was found to remain noncritical at the Anderson transition, opening the possibility for a direct superconductor-insulator transition, as seen in many experiment.

For general values of disorder $W$, and interactions $U$ and $V$, evaluating $\left[\chi_{i j}^{2}\right]_{d i s}$ is difficult, since it has to be computed with respect to the action $S_{s p}\left[c_{i}^{\dagger}, c\right]$ describing disordered interaction electrons in finite dimensions. The situation is simpler both in limits of very strong and very weak disorder, where reliable approximations are available. We first examine the limit of very strong disorder, and determine the critical value of the Fermi energy corresponding to the emergence of glassy ordering at $T=0$. To determine the transition line, to leading order in $W / U$ and $W / V$ it is sufficient to set $U=V=0$ in computing the required quantity $\chi^{(2)}$.

High disorder - Anderson transition. As the disorder grows, the system approaches the Anderson transition at $t=t_{c}(W) \sim W$. The first hint of singular behavior of $\chi^{(2)}$ in an Anderson insulator is seen by examining the deeply insulating, i. e. atomic limit $W \gg t$, where to leading order we set $t=0$ and obtain $\chi_{i j}=\delta\left(\varepsilon_{i}-\mu\right) \delta_{i j}$. We immediately find that $\chi^{(1)}(\vec{K})=\left[\delta\left(\varepsilon_{i}-\mu\right)\right]_{d i s}=P(\varepsilon=\mu)$ remains finite, but $\chi^{(2)}=\left[\delta^{2}\left(\varepsilon_{i}-\mu\right)\right]_{d i s}=+\infty$ diverges! Since we expect all quantities to behave in qualitatively the same fashion throughout the insulating phase, we anticipate $\chi^{(2)}$ to 
diverge already at the Anderson transition. Note that, since the instability of the glassy phase occurs already at $\chi^{(2)}=V^{-2}$, the glass transition must precede the localization transition. Thus, for any finite inter-site interaction $V$, we predict the emergence of an intermediate metallic glass phase separating the Fermi liquid from the Anderson insulator. Assuming that near the transition

$$
\chi^{(2)} \simeq \frac{A}{W^{2}}((t / W)-B)^{-\alpha}
$$

( $A$ and $B=t_{c} / W$ are constants of order unity), from Eq. (8) we can estimate the form of the glass transition line, and we get

$$
\delta t(W)=t_{G}(W)-t_{c}(W) \sim V^{2 / \alpha} W^{1-2 / \alpha} ; W \rightarrow \infty
$$

The glass transition and the Anderson transition lines are predicted to converge at large disorder for $\alpha<2$, and diverge for $\alpha>2$. Since all the known exponents characterizing the localization transition seem to grow with dimensionality, we may expect a particularly large metallic glass phase in large dimensions.

In order to confirm this scenario by explicit calculations, we compute the behavior of $\chi^{(2)}$ at the Anderson transition of a half-filled Bethe lattice of coordination $z=3$.We use an essentially exact numerical approach 16 based on the recursive structure of the Bethe lattice [17]. In this approach, local and non-local Green's functions on a Bethe lattice can be sampled from a large ensemble, and the compressibilities $\chi_{i j}$ can be then calculated by examining how a local charge density $n_{i}$ is modified by an infinitesimal variation of the local site energy $\varepsilon_{j}$ on another site. To do this, we have taken special care in evaluating the local charge densities $n_{i}$ by numerically computing the required frequency summations over the Matsubara axis, where the numerical difficulties are minimized. Using this method, we have calculated $\chi^{(2)}$ as a function of $W / t$ (for this lattice at half-filling $E_{F}=2 \sqrt{2} t$ ), and find that it decreases exponentially [18] as the Anderson transition is approached. We emphasize that only a finite enhancement of $\chi^{(2)}$ is required to trigger the instability to glassy ordering, which therefore occurs before the Anderson transition is reached. The resulting $T=0$ phase diagram, valid in the limit of large disorder, is presented in Fig. 1. Note that the glass transition line in this case has the form $t_{G}(W) \sim W$, in agreement with the fact that exponential critical behavior of $\chi^{(2)}$ corresponds to $\alpha \rightarrow \infty$ in the above general scenario. These results are strikingly different from those obtained in a theory which ignores localization [8], where $t_{G}(W)$ was found to be weakly dependent on disorder, and remain finite as $W \longrightarrow \infty$. Anderson localization effects thus strongly enhance the stability of the glass phase at sufficiently large disorder. Nevertheless, since the Fermi liquid to metallic glass (FMG) transition occurs at a finite distance before the localization transition, we do not

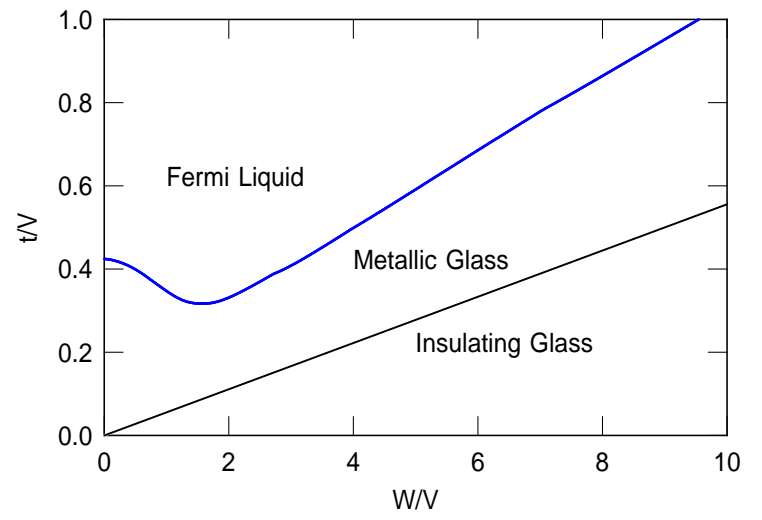

FIG. 1: Phase diagram for electrons on the $z=3$ Bethe lattice, where localization effects are treated using an exact numerical method. The results are valid in the limit of large disorder $(W / U \rightarrow \infty)$.

expect the leading quantum critical behavior [13] at the FMG transition to be qualitatively modified by the localization effects.

Low disorder - Mott transition. In the limit of weak disorder $W \ll U, V$, and interactions drive the metalinsulator transition. Concentrating on the model at halffilling, the system will undergo a Mott transition [7] as the hopping $t$ is sufficiently reduced. Since for the Mott transition $t_{M o t t}(U) \sim U$, near the transition $W \ll t$, and to leading order we can ignore the localization effects. In addition, we assume that $V \ll U$, and to leading order the compressibilities have to be calculated with respect to the action $S_{e l}$ of a disordered Hubbard model. The simplest formulation that can describe the effects of weak disorder on such a Mott transition is obtained from the dynamical mean-field theory (DMFT) 14]. This formulation, which ignores localization effects, is obtained by rescaling the hopping elements $t \rightarrow t / \sqrt{z}$ and then formally taking the limit of large coordination $z \rightarrow \infty$. To obtain qualitatively correct analytical results describing the vicinity of the disordered Mott transition at $T=0$, we have solved the DMFT equations using a 4-boson method of Kotliar and Ruckenstein [15]. At weak disorder, these equations can be easily solved in close form, and we simply report the relevant results. The critical value of hopping for the Mott transition is found to decrease with disorder, as

$$
t_{c}(W) \approx t_{c}^{o}\left(1-4(W / U)^{2}+\cdots\right),
$$

where for a simple semi-circular density of states 14] $t_{c}^{o}=3 \pi U / 64$ (in this model, the bandwidth $B=4 t$ ). Physically, the disorder tends to suppress the Mott insulating state, since it broadens the Hubbard bands and narrows the Mott-Hubbard gap. At sufficiently strong disorder $W \geq U$, the Mott insulator is suppressed even in the atomic limit $t \rightarrow 0$. The behavior of the compress- 


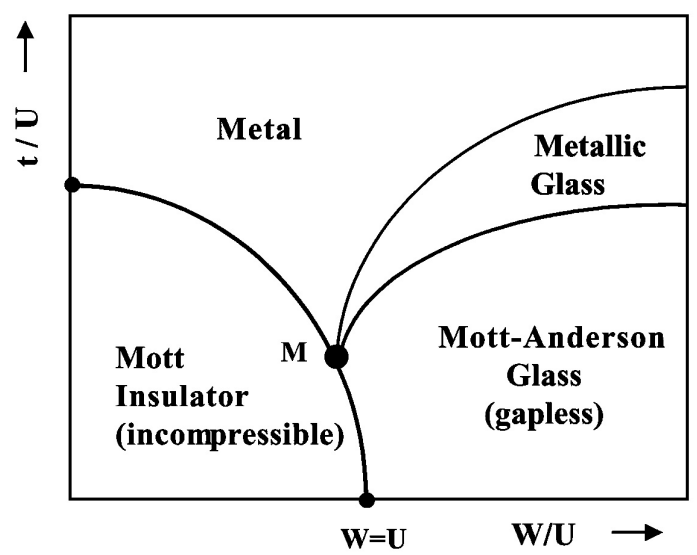

FIG. 2: Global phase diagram for the disordered Hubbard model, as a function of the hopping element $t$ and the disordered strength $W$, both expressed in units of the on-site interaction $U$. The size of the metallic glass phase is determined by the strength of the inter-site interaction $V$.

ibilities can also be calculated near the Mott transition, and to leading order we find

$$
\chi^{(2)}=\left[\frac{8}{3 \pi t_{c}^{o}}\left(1-\frac{t_{c}(W)}{t}\right)\right]^{2}\left(1+28(W / U)^{2}\right) .
$$

Therefore, as any compressibility, $\chi^{(2)}$ is found to vanish in the vicinity of the Mott transition, even in presence of finite disorder. As a result, the tendency to glassy ordering is strongly suppressed at weak disorder, where one approaches the Mott insulating state.

Finally, having analyzed the limits of weak and strong disorder, we briefly comment on what may be expected in the intermediate region $W \gtrsim U$. On general grounds, we expect a global phase diagram as shown in Fig.2. The Mott gap cannot exist for $W>U$, so in this region and for sufficiently small $t$ (i. e. kinetic energy), one enters an gapless (compressible) Mott-Anderson insulator. For $W \gtrsim U$, the computation of $\chi^{(2)}$ requires the full solution of the Mott-Anderson problem. The required calculations can and should be performed using the formulation of Ref. [16], but that difficult task is clearly beyond the scope of this letter. However, based on general arguments presented above, we expect $\chi^{(2)}$ to vanish as one approaches the Mott insulator $(W<U)$, but to diverge as one approaches the Mott-Anderson insulator $(W>U)$. Near the tetracritical point M (see Fig. 2), we may expect $\chi^{(2)} \sim \delta W^{-\alpha} \delta t^{\beta}$, where $\delta W=W-W_{M o t t}(t)$ is the distance to the Mott transition line, and $\delta t=t-t_{c}(W)$ is the distance to the Mott-Anderson line. Using this ansatz and Eq. (8), we find the glass transition line to take the form

$$
\delta t=t_{G}(W)-t_{c}(W) \sim \delta W^{\beta / \alpha} ; W \gtrsim W_{M} .
$$

We thus expect the intermediate metallic glass phase to be suppressed as the disorder is reduced, and one approaches the Mott insulating state. Physically, glassy behavior of electrons corresponds to many low-lying rearrangements of the charge density; such rearrangements are energetically unfavorable close to the (incompressible) Mott insulator, since the on-site repulsion $U$ opposes charge fluctuations. Interestingly, very recent experiments on low density electrons in silicon MOSFETs have revealed the existence of exactly such an intermediate metallic glass phase in low mobility (highly disordered) samples 4]. In contrast, in high mobility (low disorder) samples [5], no intermediate metallic glass phase is seen, and glassy behavior emerges only as one enters the insulator, consistent with our theory. Similar conclusions have also been reported in studies of highly disordered $\mathrm{InO}_{2}$ films [3] , where the glassy slowing down of the electron dynamics seems to be suppressed as the disorder is reduced and one crosses over from an Anderson-like to a Mott-like insulator. In addition, these experiments [4, 5] provide striking evidence of scale-invariant dynamical correlations inside the glass phase, consistent with the hierarchical picture of glassy dynamics, as generally emerging from mean-field approaches [11] such as the one used in this letter.

We thank S. Bogdanovich, S. Chakravarty, J. Jaroszynski, D. Popović, and Z. Ovadiyahu for useful discussions. This work was supported by the NSF grant DMR-9974311 and the National High Magnetic Field Laboratory.

[1] P.A. Lee and T. V. Ramakrishnan, Rev. Mod. Phys. 57, 287 (1985).

[2] M. Ben-Chorin, et al., Phys. Rev. B 48, 15025 (1993); Z. Ovadyahu and M. Pollak, Phys. Rev. Lett. 79, 459 (1997).

[3] A. Vaknin et al., Phys. Rev. Lett. 81, 669 (1998).

[4] S. Bogdanovich and D. Popović, Phys. Rev. Lett. (2002, in press), cond-mat/0106545

[5] J. Jaroszynski, Dragana Popović, and T. M. Klapwijk, cond-mat/0205226

[6] A. L. Efros et al., J. Phys. C 8 L49 (1975); J. H. Davies, et al., Phys. Rev. Lett. 49, 758 (1982); Phys. Rev. B 29, 4260 (1984); M. Pollak, Philos. Mag. B 50, 265 (1984); M. Pollak and M. Ortuño, Sol. Energy Mater. 8, 81 (1982).

[7] N. F. Mott, Metal-Insulator Transitions (Taylor and Francis, London 1974).

[8] A. A. Pastor and V. Dobrosavljević, Phys. Rev. Lett. 83, 4642 (1999).

[9] P. W. Anderson, Phys. Rev. 109, 1498 (1958).

[10] Recent work 8] has shown that in presence of random site energies, such random interactions are generated by renormalization even if one starts with purely repulsive (e. g. Coulomb) interactions in the bare Hamiltonian. Similar conclusions have been obtained numerically in: 
E. R. Grannan and C. C. Yu, Phys. Rev. Lett. 71, 3335 (1993).

[11] K. H. Fischer and J. A. Hertz, Spin Glasses (Cambridge University Press, Cambridge, England, 1993).

[12] A. Kapitulnik and G. Kotliar, Phys. Rev. Lett. 54, 473476 (1985).

[13] D. Dalidovich and V. Dobrosavljević, preprint cond-mat/0205269

[14] A. Georges et al. , Rev. Mod. Phys. 68, 13 (1996).
[15] G. Kotliar and A. E. Ruckenstein, Phys. Rev. Lett. 57, 1362-1365 (1986).

[16] V. Dobrosavljevic and G. Kotliar, Phys. Rev. Lett. 78, 3943 (1997).

[17] R. Abou-Chacra, P. W. Anderson, D. J. Thouless, J. Phys. C 6, 1734 (1973).

[18] A. D. Mirlin, Y. N. Fyodorov, Nucl. Phys. B 366, 507 (1991). 